\title{
Percepção do aluno sobre sua interação com o professor e status sociométrico
}

\author{
Student's perception of the teacher-student \\ relationship and sociometric status
}

\author{
Selma de Cássia MARTINELLI \\ Andreza SCHIAVONI'
}

\begin{abstract}
Resumo
Esta pesquisa teve por objetivo verificar a relação entre a percepção de estudantes sobre as expectativas de seus professores a seu respeito e o seu status sociométrico, investigado por meio das escolhas do grupo de amigos. Participaram do estudo 130 alunos do ensino fundamental, de ambos os sexos, entre 9 e 10 anos de idade, de duas escolas públicas do interior do Estado de São Paulo. A percepção dos participantes foi obtida por meio de uma escala contendo vinte afirmações, onze positivas, que indicam boa percepção do aluno, e nove que indicam uma percepção negativa. Para avaliar o status sociométrico foi solicitado aos participantes que indicassem três nomes de colegas da classe com quem gostariam de estudar, de brincar, de não estudar e de não brincar. Os resultados indicaram que os alunos que acreditam que seu professor tem uma percepção positiva sobre eles também obtiveram médias maiores de aceitação por seu grupo de amigos. Os participantes que demonstraram uma percepção de expectativas negativa também foram mais rejeitados por seus pares. O estudo aponta para a necessidade de se considerar as relações sociais estabelecidas na escola e promove uma reflexão sobre a importância dessas relações no contexto do processo ensino-aprendizagem.
\end{abstract}

Unitermos: Interação professor-aluno. Medidas perceptivas. Técnicas sociométricas.

\begin{abstract}
The aim of this study was to verify the relationship between students' perceptions about their teachers' expectations of them and their sociometric status, investigated through the selection of groups of friends. Participants comprised 130 male and female elementary school children, aged between 9 and 10, in two public schools from the São Paulo state interior. Participants' perception was measured via a scale consisting of twenty statements, of which eleven were positively, and nine negatively, stated. To assess sociometric status, participants were required to indicate three classmates with whom they would like to study and to play, and three with whom they would not like to study and to play. Results showed that those students who believed that their teacher had a positive perception towards them also had a higher average peer group acceptance. Those participants who demonstrated a negative perception of expectations were the ones most rejected by their peers. This study points to the importance of the social relationships established at school and encourages a reflection on the importance of these relationships in the context of the teach/learn process.
\end{abstract}

Uniterms: Teacher-student interaction. Perceptual measures. Sociometric techniques.

$\boldsymbol{\nabla \nabla \nabla \nabla}$

1 Universidade Estadual de Campinas, Departamento de Psicologia Educacional, Grupo de Estudos e Pesquisa em Psicopedagogia. R. Bertrand Russell, 801, Cidade Universitária, Barão Geraldo, 13081-970, Campinas, SP, Brasil. Correspondência para/Correspondence to: S.C. MARTINELLI. E-mail: <selmacm@unicamp.br>. 
Dentre diferentes possibilidades de relacionamento interpessoal em sala de aula, observa-se que a formação de expectativas e percepções pelas partes envolvidas permeia qualquer que seja a relação estabelecida. Assim, professores baseiam suas atitudes nas percepções e expectativas que têm sobre seus alunos que, por sua vez, criam suas próprias maneiras de perceberem seus professores, a si mesmos e a seus colegas.

Os resultados de uma pesquisa de grande repercussão na década de 1960, feita por Rosenthal e Jacobson (1968), atentaram para a presença inevitável da formação de expectativas de professores sobre os alunos no processo de ensino e aprendizagem. Por considerarem como principal fonte de expectativas do professor o desempenho dos alunos, foram apresentadas aos professores envolvidos no estudo as crianças que tinham maior probabilidade de melhorar seu rendimento, pelo fato de terem sido as que obtiveram os melhores resultados em um teste de inteligência. Contudo, tais alunos foram selecionados aleatoriamente sem que realmente demonstrassem um maior QI total no teste. Estas crianças, cujo melhor desenvolvimento intelectual era esperado, mostraram ao final do ano letivo uma aprendizagem mais eficiente e foram descritas pelos professores como as que mais provavelmente teriam sucesso no futuro, as mais interessadas, as que apresentavam maior curiosidade intelectual e as mais felizes.

Após a divulgação do trabalho de Rosenthal e Jacobson (1968) outros estudos foram realizados, cujos resultados continuaram apontando para a influência das expectativas e percepções de professores sobre os alunos (Babad, 1995; Babad, Inbar \& Rosenthal, 1982; Brophy \& Good, 1974; Fuchs, 1994; Herbert, 1992; Hiebert, 1982; McLeod, 1994; Meltzer, Katzir-Cohen, Miller \& Roditi, 2001; Rolison \& Medway, 1985). Estudos voltados para a análise da expectativa de professores e sua relação com o desempenho acadêmico ou com dificuldades de aprendizagem também podem ser encontrados (Bear, Minke, Griffin \& Deemer, 1998; Davidson \& Lang, 1960; Schiavoni \& Martinelli, 2005). As conclusões gerais desses estudos têm revelado que as percepções das crianças a respeito dos sentimentos de seus professores para com elas estão correlacionadas com o desempenho acadêmico. De maneira geral, apontam para o fato de que as 328 crianças que acreditam que seus professores têm uma percepção mais negativa sobre elas têm apresentado baixo desempenho acadêmico ou dificuldades de aprendizagem em uma ou mais áreas do conhecimento.

Segundo Brophy e Good (1974), um importante determinante das expectativas do professor são as características dos alunos, sejam individuais ou grupais. Dentre essas características, nota-se como fonte de formação de expectativas as diferenças entre grupos sociais, etnia, sexo, desempenho acadêmico, personalidade, aparência física, características da fala e clareza na escrita, e até o local onde o aluno senta na classe.

Ao mesmo tempo em que se formam percepções e expectativas por parte do professor, o aluno passa pelo mesmo processo. Em razão da qualidade do ensino ministrado pelo professor, das suas características individuais e da relação estabelecida com o aprendiz, percepções e expectativas sobre o professor também se estabelecem. Assim, expectativas, percepções e atitudes tanto de professores em relação aos alunos quanto destes sobre aqueles podem influenciar a interação (Brophy \& Good, 1974).

Da mesma forma que a interação professor-aluno pode ser influenciada por percepções, expectativas e atitudes, a interação entre pares também é estabelecida em função dessas variáveis. Na idade escolar, os colegas passam a representar uma nova fonte de relações, com novas possibilidades de interações, que geralmente vêm acompanhadas do desejo de aceitação no grupo. Segundo Newcombe (1999), para participar adequadamente de um grupo é exigido um grau de aceitação por parte de seus membros. Na dinâmica da sala de aula, pode-se observar que alguns alunos são mais aceitos que outros, e que geralmente esses são os que demonstram grande número de competências cognitivas e sociais, o que pode resultar em comportamentos mais amigáveis e afetuosos, além de formas mais eficazes de interação. Para o autor, alunos menos aceitos por seus pares apresentam um padrão de comportamento característico, que inclui conflitos, ser alvo de provocações, brigas, discussões e agressões, imaturidade, habilidades sociais e cognitivas reduzidas.

Conforme assinalam Sisto e Martinelli (2006), a criança que apresenta dificuldades em desempenhar bem suas funções sociais está sujeita a sofrer consequências emocionais que podem resultar em uma baixa autoestima ou mesmo em uma percepção negativa de 
si mesma. A criança que se percebe como não querida ou adequada pode, por sua vez, adotar um padrão de comportamento que altere suas relações com os companheiros.

Estudos têm se preocupado em identificar a posição sociométrica que as pessoas ocupam nos grupos aos quais pertencem. A maioria deles realiza essa medida por meio de técnicas sociométricas de escolha. Dentre elas, a mais utilizada é o teste sociométrico que, segundo Moreno (1972), é um instrumento que serve para medir e analisar a organização dos grupos sociais, além de permitir determinar a posição de cada indivíduo no grupo em que estuda, vive ou trabalha.

No que se refere à avaliação sociométrica de alunos, pesquisas têm investigado esse status para verificar a rede de amizades dos mais populares e dos mais rejeitados, a relação entre indicadores sociométricos e características físicas, comportamentais e emocionais, a relação entre esses indicadores e problemas de atenção e a relação com o desenvolvimento cognitivo e a aprendizagem (ou com problemas de aprendizagem).

Ray (1995) observou a posição sociométrica de um grupo de crianças em situações de sala de aula e em suas atividades lúdicas. Encontrou que, em ambos os contextos, as crianças populares têm mais amigos, enquanto as mais rejeitadas demonstram um ciclo bem menor de amizade.

Estudos como o de Cantrell e Prinz (1985), Bierman (1987) e Sabornie (1987) averiguaram se a baixa popularidade estaria associada a problemas de comportamento e encontraram que os sujeitos com altos níveis de rejeição, apontados pelo teste sociométrico, apresentavam problemas de adequação de comportamento na escola. Os trabalhos de Bierman, Smoot e Aumiller (1993) e Coie, Terry, Lenox e Lochman (1995) concluíram que metade das crianças fisicamente agressivas que frequentavam a escola elementar era rejeitada por seus pares.

Adotando outra perspectiva, Sisto (2003) investigou como estudantes do ensino fundamental que foram rejeitados nas escolhas de seus amigos para estudar percebiam-se em termos de agressividade. Os estudantes mais aceitos declararam ter menos condutas agressivas, embora nem todas as crianças agressivas tenham sido rejeitadas. Em estudo mais recente, Sisto (2005) verificou a relação entre aceitação e rejeição em situação de estudo e a percepção de agressividade por crianças em dois contextos diferentes, familiar e escolar, e encontrou correlações significativas entre as medidas sociométricas e de agressividade, com indicações de que quanto maior a aceitação social, menor a agressividade declarada pelos estudantes.

Morais, Otta e Scala (2001) pesquisaram as correlações entre escolhas sociométricas positivas e negativas e atribuição de características comportamentais por parte de companheiros de uma classe de pré-escola. Foram pesquisadas características comportamentais na esfera social, aceitação/isolamento social, participação/não participação nas atividades, dependência/independência da professora e dominância/submissão; e, no âmbito afetivo, tristeza/alegria e medo/coragem. Verificou-se que as crianças associaram as escolhas positivas a atributos social e afetivamente positivos.

Lee-Manoel, Morais, Bussab e Otta (2002) investigaram a relação entre julgamentos de atratividade física, indicadores sociométricos e atributos comportamentais em crianças de cinco anos em média. Por entenderem que, mesmo antes dessa idade, os companheiros se tornam, além dos adultos, importantes elementos de referência de aceitação social, a hipótese era de que relações positivas entre autoconceito e aceitação social e entre autoavaliação positiva e atratividade física seriam encontradas. A atratividade das crianças foi avaliada por adultos familiarizados com elas, por adultos que não as conheciam, por seus colegas e por elas próprias. Foram apuradas as escolhas positivas e negativas que cada criança recebeu para as dimensões alegre/triste, agressivo/não agressivo, sociável/isolado e colaborador/perturbador. Correlações significativas foram encontradas entre a avaliação de atratividade física segundo os adultos familiarizados e os colegas com escolhas positivas, e com atributos comportamentais pró-sociais.

Toneloto (2002) teve como objetivo identificar crianças entre seis e nove anos desatentas no contexto de sala de aula e verificar como percebem e são percebidas por seus colegas. A presença de um número maior de atitudes negativas em relação aos colegas e à escola 
no grupo de crianças com problemas de atenção foi observada. Verificou-se, também, que esse grupo foi o menos aceito pelos colegas de sala de aula, sendo seus integrantes percebidos como os menos populares. Para a autora, a falta de atenção por si já pode causar grandes problemas para a aprendizagem, e isso parece se potencializar quando associado a uma interação entre pares prejudicada.

As investigações sobre a avaliação sociométrica de alunos também têm se preocupado em relacioná-la ao desenvolvimento cognitivo e à aprendizagem ou a problemas de aprendizagem. Sisto, Urquijo e Souza (1999) investigaram as relações entre desenvolvimento cognitivo medido por provas de imagem mental, provas operatórias de conservação de massa e comprimento, construção de possíveis em equidistância, arranjos de dados e teste sociométrico. Os resultados indicaram que crianças com alta aceitação por seus pares apresentaram um desenvolvimento cognitivo geral superior ao das crianças com alta rejeição. Outros estudos, como os de Badami e Badami (1975), Horowitz (1981), Coben e Zigmond (1986), Ackerman e Hawes (1986) e Stone e La Greca (1990), entre outros, também encontraram relações significativas entre baixa popularidade, dificuldades de aprendizagem e baixo desempenho acadêmico.

Stiliadis e Wiener (1989) verificaram que crianças que apresentavam dificuldades de aprendizagem obtiveram escores de percepção social mais baixos e menor aceitação social por seus pares. Dias (2003), por sua vez, investigou as relações entre a aprendizagem mediada pelo conflito sociocognitivo e o grau de percepção de crianças de cinco a sete anos sobre sua posição sociométrica no grupo. Ao considerar a percepção como um todo, encontrou uma relação entre maior percepção e melhor aprendizagem.

Saravali (2004) realizou um estudo que teve por objetivo investigar a posição sociométrica que crianças de quarta série, apontadas pela professora como alunos com dificuldades de aprendizagem, ocupavam no grupo ao qual pertenciam. Avaliou-se também a relação entre a posição sociométrica e os dados obtidos pela análise do histórico escolar desses alunos. Os resultados apontaram para baixa ou nenhuma escolha por parte dos colegas para as crianças com queixa de dificuldade de aprendizagem. Observou-se, ainda, que o julgamento da professora sobre o aluno com dificuldades de aprendizagem tem relação direta com o número de escolhas que ele recebe no teste sociométrico. Todas as crianças apontadas pela professora foram pouco escolhidas nos critérios afetivo e intelectual. Verificouse, por fim, que as crianças que tinham os piores conceitos no histórico escolar tiveram também menos escolhas no teste do que as outras crianças com dificuldade de aprendizagem que tinham conceitos um pouco melhores.

A sugestão de que percepções e expectativas estão presentes na interação entre pares e também na interação professor-aluno, como dito anteriormente, pode ser confirmada pelos resultados encontrados por Moreno (1972). Em uma pesquisa realizada com alunos de uma escola que oferecia turmas desde o jardim da infância até o ensino fundamental, o pesquisador verificou tanto a posição sociométrica dos grupos de alunos quanto a relação entre os julgamentos dos professores e as escolhas dos colegas. Como resultado da avaliação sociométrica, pôde perceber que a estrutura organizacional do grupo era muito mais complexa do que se supunha: havia alunos muito escolhidos, pouco escolhidos e constituições recíprocas bastante diversificadas. A relação investigada entre julgamento do professor e escolha dos pares foi possível por meio de um teste de opinião aplicado aos professores. Neste, deveriam escrever o nome dos alunos que acreditavam que receberiam o maior e o menor número de escolhas por parte dos colegas. Em grande parte dos casos, os resultados obtidos no teste com os professores coincidiram com as escolhas feitas entre os colegas.

Com base nos estudos apresentados, nota-se que a interação entre pares tem sido pesquisada sob diferentes enfoques. Observa-se, também, que a preocupação mais comum é relacioná-la a uma outra variável, como problemas de atenção, aprendizagem, dificuldades de aprendizagem. A relação entre o julgamento do professor sobre os alunos considerados mais e menos aceitos e as reais escolhas dos colegas, como foi investigada por Moreno (1972) e Saravali (2004), também representa um caminho interessante para se estudar a interação.

A análise da percepção do aluno sobre o que imagina ser o julgamento do professor a seu respeito e sua aceitação ou rejeição por parte dos colegas, verificada por meio do teste sociométrico, não foi 
encontrada na literatura, e também parece ser um caminho bastante revelador das relações estabelecidas no seio da instituição escolar. Assim, com o intuito de observar o olhar do aluno sobre aspectos envolvidos nas interações presentes em sala de aula, este estudo objetivou verificar se a percepção que o aluno revela sobre a expectativa do professor a seu respeito apresenta relações com a aceitação e a rejeição de seus colegas nas situações de estudar e brincar na escola.

\section{Método}

\section{Participantes}

Participaram desta pesquisa, realizada no ano de 2005, 130 alunos entre nove e dez anos de idade, 67 do sexo masculino e 63 do sexo feminino, que frequentavam a terceira série do ensino fundamental, de duas escolas públicas estaduais das cidades de São Carlos e Araras, no Estado de São Paulo.

\section{Instrumentos}

Medida de avaliação da relação professor-aluno: Trata-se de uma escala de avaliação da percepção de alunos sobre as relações estabelecidas com o professor, elaborada por Martinelli (2005). A análise fatorial exploratória do instrumento evidenciou uma estrutura de seis fatores, que explicou 58,71\% da variância total. A análise da consistência interna das dimensões obtidas evidenciou valores de alfa de Cronbach adequados, com um valor de 0,8456 para o total da medida. O estudo da sua estabilidade temporal, por meio do método teste-reteste, também evidenciou valores de correlação elevados (0,788).

A escala contém vinte afirmações, sendo que onze delas indicam uma percepção positiva do aluno, enquanto as outras nove indicam uma percepção negativa por parte do mesmo. Abaixo de cada assertiva são propostas três alternativas de respostas que indicam a frequência de ocorrência dessas situações (sempre, às vezes e nunca). Para as questões positivas do instrumento foram atribuídas a pontuação 2 para a resposta sempre, 1 para às vezes e 0 para nunca. Às negativas foram atribuídos os valores inversos: 0 para a resposta sempre, 1 para às vezes e 2 para nunca, o que revelou uma pontuação bruta para cada sujeito que variava de 0 a 40 pontos.
Medida de avaliação sociométrica:Trata-se de um instrumento com quatro questões: duas referentes a estudar e duas a brincar. Nelas, os participantes deveriam indicar três nomes de colegas da classe com quem gostariam de estudar, três com quem não gostariam de estudar, três com quem gostariam de brincar e mais três com quem não gostariam de estudar. Para as questões referentes à aceitação (estudar e brincar) foram atribuídas as pontuações 3 para a primeira escolha, 2 para a segunda e 1 para a terceira. Valores negativos foram atribuídos às questões relativas à rejeição (não estudar e não brincar), sendo, então, -3 para a primeira escolha, -2 para a segunda e -1 para a terceira. Uma soma dos valores positivos e dos valores negativos foi feita para cada participante, o que resultou em uma medida de aceitação e outra de rejeição.

\section{Procedimentos}

O estudo foi iniciado após o consentimento da equipe gestora, dos professores e dos pais dos alunos das escolas contatadas para a realização da pesquisa, de acordo com o definido na Resolução 196/96, e após trazerem o Termo de Consentimento Livre e Esclarecido assinado pelos pais em duas vias. Somente integraram a pesquisa as crianças cujos pais autorizaram a participação. Nesse documento constavam informações acerca dos instrumentos que foram administrados, bem como dos objetivos da pesquisa.

No procedimento de coleta de dados as pesquisadoras reuniram grupos de dez alunos por vez para a aplicação dos instrumentos, que ocorreu em horário regular de aula, nas bibliotecas das escolas. A ordem de aplicação dos instrumentos foi sorteada: iniciou-se com o teste sociométrico e, em seguida, escala de percepção; a ordem foi a mesma para todos os estudantes que realizaram as atividades em um único dia, com duração aproximada de quarenta minutos. A leitura da escala de percepção foi feita pela experimentadora responsável, que lia a primeira questão e aguardava o preenchimento dos alunos e assim sucessivamente, até o final do instrumento.

\section{Resultados}

Os resultados serão apresentados em duas etapas. Em primeiro lugar, os resultados da análise 
estatística descritiva da amostra em relação às duas variáveis investigadas serão analisados; em seguida, serão apresentadas as análises com base em uma prova de correlação de Spearman, para verificar a relação entre as duas variáveis, e análises para verificar as diferenças entre os grupos e localizar as diferenças significativas encontradas no que diz respeito à percepção dos alunos sobre as expectativas de seus professores a seu respeito e ao status sociométrico dos participantes.

\section{Percepção de expectativas}

A média encontrada na escala de percepção de alunos sobre as expectativas de professores a seu respeito foi de 29,48 pontos, com desvio-padrão igual a 7,05 . Dentre os grupos de alunos estabelecidos segundo os níveis de percepção de expectativas (Tabela 1), observou-se um número maior de participantes com uma percepção moderada, cujo grupo apresentou média igual a 30,51 e desvio-padrão igual a 2,49.

\section{Status sociométrico - aceitação e rejeição}

Na medida de aceitação dos participantes foram encontradas pontuações que variaram de 0 a 84, com média de 10,96 e desvio-padrão de 11,46, indicando que algumas crianças não foram escolhidas para brincar e para estudar por nenhum dos colegas, enquanto outras foram escolhidas por vários colegas. Na medida de rejeição, as pontuações variaram de 0 a 53, com média de 9,38 e desvio-padrão de 10,89, indicando que, da mesma forma, enquanto algumas crianças não foram rejeitadas na escolha de seus colegas, outras foram altamente rejeitadas.

\section{Percepção de alunos e status sociométrico}

A análise de correlação revelou que a criança que acredita que seu professor tem uma percepção positiva sobre ela também obteve médias maiores de aceitação por seu grupo de amigos, apresentando um coeficiente de correlação (rs) de 0,315 e nível de significância $(p=0,0001)$. Por sua vez, a percepção negativa da criança esteve correlacionada com sua rejeição pelo grupo ( $r s=0,352$ e $p<0,0001)$. Na Tabela 2 encontram-se discriminadas as diferenças de média em aceitação e rejeição em função da percepção da criança.

Os resultados apresentados (Tabela 2) indicam que o grupo de alunos que revelou acreditar que o seu professor tinha uma percepção positiva a seu respeito também obteve médias de aceitação pelos colegas mais alta que os outros grupos, enquanto o grupo que demonstrou uma percepção mais negativa em relação à opinião do professor sobre si também teve uma média de rejeição mais alta que os demais grupos.

Tabela 1. Distribuição dos participantes segundo os grupos de percepção sobre os sentimentos de seus professores sobre eles. São Carlos (SP) e Araras (SP), 2005.

\begin{tabular}{lllll}
\cline { 2 - 5 } & Percepção de alunos & n & Média & Desvio-padrão \\
\cline { 2 - 5 } & Grupo 1 - percepção negativa & 38 & 20,58 & 4,61 \\
Grupo 2 - percepção moderada & 55 & 30,51 & 2,49 \\
Grupo 3 - percepção positiva & 37 & 37,08 & 1,50 \\
\cline { 2 - 5 } & Total & 130 & 29,48 & 7,05 \\
\cline { 2 - 5 }
\end{tabular}

Tabela 2. Médias de aceitação e rejeição em função dos grupos de crianças e suas percepções sobre os sentimentos de seus professores sobre elas. São Carlos (SP) e Araras (SP), 2005.

\begin{tabular}{lcccc}
\hline Percepção & Média de aceitação & Desvio-padrão & Média de rejeição & Desvio-padrão \\
\hline Grupo 1 - percepção negativa $(n=38)$ & 6,84 & 6,18 & 14,08 & 13,04 \\
Grupo 2 - percepção moderada $(n=55)$ & 9,93 & 9,52 & 8,55 & 9,54 \\
Grupo 3 - percepção positiva $(n=37)$ & 16,73 & 15,54 & 5,78 & 8,71 \\
\hline Total & 10,96 & 11,46 & 9,38 & 10,89 \\
\hline
\end{tabular}


Tabela 3. Incidência média, valores de U, níveis de significância e comparação entre os diferentes grupos de crianças e suas percepções sobre os sentimentos de seus professores sobre elas e a aceitação entre os pares. São Carlos (SP) e Araras (SP), 2005.

\begin{tabular}{lcccc}
\hline Grupos pelo critério de percepção & Valores de $U$ & $Z$ & Valores de $p$ & Comparação entre os grupos \\
\hline G1 e G2 & 863,50 & $-1,422$ & 0,155 & NS \\
G2 e G3 & 686,50 & $-2,639$ & 0,008 & $\mathrm{G} 3>\mathrm{G} 2^{*}$ \\
G1 eG3 & 331,00 & $-3,948$ & 0,000 & $\mathrm{G} 3>\mathrm{G} 1^{*}$ \\
\hline
\end{tabular}

*Valores significativos; U e Z: indicadores da prova de Mann-Whitney; NS: não significativo.

Tabela 4. Incidência média, valores de U, níveis de significância e comparação entre os diferentes grupos de crianças e suas percepções sobre os sentimentos de seus professores sobre elas e a rejeição entre os pares. São Carlos (SP) e Araras (SP), 2005.

\begin{tabular}{lcccc}
\hline Grupos pelo critério de percepção & Valores de $U$ & $Z$ & Valores de $p$ & Comparação entre os grupos \\
\hline G1 e G2 & 779,50 & $-2,081$ & 0,037 & $\mathrm{G}_{1>\mathrm{G} 2^{*}}$ \\
G2 e G3 & 757,50 & $-2,085$ & 0,037 & $\mathrm{G} 2>\mathrm{G} 3^{*}$ \\
G1 e G3 & 389,00 & $-3,346$ & 0,001 & $\mathrm{G} 1>\mathrm{G} 3^{*}$ \\
\hline
\end{tabular}

* Valores significativos; U e Z: indicadores da prova de Mann-Whitney.

As diferenças de média encontradas entre os grupos, com relação à aceitação de seus pares, foram analisadas pelo teste de Kruskall-Wallis, que revelou serem significativas as diferenças encontradas $(p<0,0001)$. A direção destas diferenças, verificada pelo teste de Mann-Whitney, estão na Tabela 3.

Os resultados do teste de Mann-Whitney indicaram diferenças significativas entre os grupos G3 e G2 e G3 e G1, revelando que os participantes que declararam perceber-se de forma mais positiva por seus professores também foram mais aceitos por seus pares, sendo esta significância maior entre os grupos extremos. Não foi encontrada diferença significativa entre os grupos com percepção moderada (G2) e os com percepção negativa (G3).

O resultado do teste de Kruskall-Wallis, apresentado na Tabela 2, com relação à rejeição, também revelou significância estatística $(p=0,002)$. A direção destas diferenças, verificada pelo teste de Mann-Whitney, encontra-se discriminada na Tabela 4.

Os resultados do teste de Mann-Whitney indicaram diferenças significativas entre todos os grupos, revelando que os participantes que declararam perceber-se de forma mais negativa por seus professores também foram mais rejeitados por seus pares.

\section{Discussão}

Os resultados deste estudo, somados a outros, reafirmam a importância de se estudar e verificar como o funcionamento afetivo pode se relacionar com a vida social e escolar de crianças e adolescentes. A maneira como a criança se percebe no contexto da escola e em relação aos seus professores e amigos é refletida, de forma indireta, em seu comportamento, na forma de se estimar, bem como de se sentir estimada e valorizada pelos outros.

Estudos que têm priorizado a informação de sentimentos por parte de crianças e adultos têm sido bastante utilizados, tendo em vista que sentimentos de autoestima, tristeza, autoconfiança, entre outros, nem sempre são observados com facilidade por pessoas externas. Assim, os instrumentos que revelam a autopercepção do indivíduo têm sido cada vez mais propostos, na tentativa de se conhecer mais acerca de seus sentimentos. Neste estudo o instrumento de percepção usado buscou conhecer a opinião da criança e como ela se percebe do ponto de vista do olhar do professor. As assertivas envolviam basicamente situações de sala de aula, e seu conteúdo pautava-se na aprovação e reprovação por parte do professor a respeito do comportamento e desempenho do aluno, sentimentos de 
apreço ou de desvalorização, bem como de aproximação ou afastamento em relação a ele, o que permite observar o quanto a criança se sente acolhida ou rejeitada nessa relação. Os resultados revelaram que, de maneira geral, as crianças manifestaram uma percepção mais positiva de suas relações com o professor do que negativa, mas indicaram também que, para alguns alunos, esta percepção apresentou-se muito negativa, enquanto, para outros, mostrou-se muito favorável.

A busca de correlações entre essas percepções e a aceitação ou rejeição dos alunos em relação aos amigos permitiu verificar como essas situações apareceram colocadas neste grupo. Embora medidas dessa natureza não possam ser generalizadas, por tratarem de uma informação pontual e contextualizada, elas parecem demonstrar que, neste caso, e isso talvez possa estar presente em outras situações, quanto mais a criança se percebeu rejeitada e pouco valorizada pelo professor, maior foi a rejeição de seus colegas nas situações que envolviam a escolha para estudar e brincar. Por outro lado, quanto mais positiva a percepção da criança, mais aceita foi pelos colegas para estudar e brincar. Embora não se tenha verificado diferenças estatisticamente significativas entre o grupo de percepção negativa e percepção moderada, o grupo com percepção negativa teve menor aceitação que os demais. A ausência de significância estatística neste caso não desfaz a tendência verificada no estudo.

Embora os estudos encontrados na literatura não tenham avaliado as mesmas relações propostas nesta pesquisa, tem-se encontrado evidências de que a maior ou menor aceitação da criança pelo grupo está associada a fatores comportamentais (Bierman, 1987; Cantrell \& Prinz, 1985; Morais et al., 2001; Sabornie, 1987), agressividade (Bierman et al., 1993; Coie et al., 1995; Sisto, 2003), julgamentos de atratividade física (Lee-Manoel et al., 2002) e ao próprio desempenho acadêmico (Badami \&Badami, 1975; Horowitz, 1981; Coben \& Zigmond, 1986), entre outros. Esses dados revelam que a maneira como as crianças são percebidas pelos outros ou, como neste estudo, como elas se veem na opinião dos outros, tem se mostrado relacionada à sua maior ou menor aceitação no grupo de amigos.

Embora esses estudos tenham conseguido apontar algumas relações encontradas entre as aceitações ou rejeições e fatores comportamentais, acredita- -se que ainda seja prematuro elaborar conclusões a esse respeito, uma vez que as investigações têm seguido caminhos diferentes para a avaliação desses aspectos, além do fato de que nem sempre essas relações foram comprovadas em sua totalidade, como foi o caso do estudo aqui descrito. Desta forma, sugere-se que outras investigações que contemplem essas mesmas variáveis possam ser realizadas, inclusive com um maior número de participantes, já que, neste estudo, o grupo de participantes foi restrito.

\section{Considerações Finais}

Esses resultados remetem a algumas considerações. Os mais diferentes teóricos do desenvolvimento humano têm sido unânimes em afirmar que a fase escolar, além de ser importante pelo progresso cognitivo que se desencadeia, é também o momento em que as relações sociais se veem ampliadas e ganham importância na vida da criança. Nesta fase, as relações de amizade que se estabelecem atuam como a base das relações sociais para a criança. Da mesma forma, a figura do professor como o modelo adulto que se apresenta como externo à sua família torna-se presente, visto ser com quem a criança mediará as relações com a escola e com o conhecimento.

Uma criança que não tem sucesso na escola vai acumulando experiências de fracasso sucessivamente, o que pode levá-la a se perceber negativamente em relação aos demais. Sua aprendizagem pode se tornar mais prejudicada à medida que tem dificuldade para fazer amigos, relacionar-se e, aos poucos, pode ficar à margem do grupo, formando uma imagem deformada de si mesma.

As investigações apresentadas neste estudo apontaram para a existência de relação entre a posição sociométrica de alunos e características físicas, comportamentais, emocionais, problemas de atenção, problemas de aprendizagem, relação professor-aluno, entre outras. Nesse sentido, os dados obtidos com todas essas pesquisas sugerem uma reflexão para todos os envolvidos no processo de ensino e aprendizagem, para que estejam atentos a todas as variáveis presentes no contexto escolar e para que possam intervir de modo a favorecer o processo ensino-aprendizagem. 


\section{Referências}

Ackerman, D., \& Hawes, C. (1986). Sociometric status and after-school social activity of children with learning disabilities. Journal of Learning Disabilities, 19 (7), 416-419.

Babad, E. Y. (1995). The teachers' pet phenomenon, students' perceptions of teachers' differential behavior, and students' morale. Journal of Educational Psychology, 87 (3), 361-374.

Babad, E., Inbar, J., \& Rosenthal, R. (1982). Pygmalion, galatea, and the golem: investigations of biased and unbiased teachers. Journal of Educational Psychology, 74 (4), 459-474.

Badami, H., \& Badami, C. (1975). A study of group status in relation to school achievement as revealed by a sociometric test. Journal of Psychological Researches, 19 (1), 24-28.

Bear, G. C., Minke, K. M., Griffin, S. M., \& Deemer, S. A. (1998). Achievement-related perceptions of children with learning disabilities and normal achievement: group and developmental differences. Journal of Learning Disabilities, 31 (1), 91-104.

Bierman, K. (1987). The clinical significance and assessment of poor peer relations: Peer neglect versus peer rejection. Journal of Developmental and Behavioral Pediatrics, 8 (4), 233-240.

Bierman, K. L., Smoot, D. L., \& Aumiller, K. (1993). Characteristics of aggressive-rejected, aggressive (nonrejected), and rejected (non-aggressive) boys. Child Development, 64 (1), 139-151.

Brophy, J.E., \& Good, T. L. (1974). Teacher-studentrelationships: cause and consequences. New York: Holt, Rinehart and Winston.

Cantrell, V., \& Prinz, R. (1985). Multiples perspectives of rejected, neglected, and accepted children: Relation between sociometric status and behavioral characteristics. Journal of Consulting and Clinical Psychology, 53 (6), 884-889.

Castro, R. E. F., Melo, M. H. S., \& Silvares, E. F. M. (2003). O julgamento de pares de crianças com dificuldades interativas após um modelo ampliado de intervenção. Psicologia: Reflexão e Crítica, 16 (2), 309-318.

Coben, S., \& Zigmond, N. (1986).The social integration of learning disabled students from self contained to mainstream elementary school settings. Journal of Learning Disabilities, 19 (10), 614-618.

Coie, J. D., Terry, R., Lenox, K., \& Lochman, J. (1995). Childhood peer rejection and aggression as predictors of stable patterns of adolescent disorder. Development and Psychopathology, 7(4), 697-713.

Davidson, H., \& Lang, G. (1960). Children's perceptions of their teachers' feelings toward them related to self-perception, school achievement and behavior. Journal of Experimental Education, 29 (2), 107-118.
Dias, F. (2003). Percepção social e cognição em situações de aprendizagem por conflito sociocognitivo. PsicoUSF, 8 (1), 47-52.

Fuchs, L. S. (1994). The relation between teachers' beliefs about the importance of good student work habits, teacher planning, and student achievement. Elementary School Journal, 94 (3), 331-345.

Herbert, J. M. (1992). Intervening to influence teachers' actions and students' involvement. Journal of Personnel Evaluation in Education, 6 (3), 249-261.

Hiebert, B. (1982). Affective influences on learning disabled adolescents. Learning Disability Quarterly, 5 (4), 334-343.

Horowitz, E. (1981). Popularity, decentering ability, and role taking skills in learning disabled and normal children. Learning Disability Quarterly, 4 (1), 23-30.

Lee-Manoel, C. L., Morais, M. L. S., Bussab, V. S. R., \& Otta, E. (2002). Quem é bom (e eu gosto) é bonito: Efeitos da familiaridade na percepção de atratividade física em pré-escolares. Psicologia Reflexão e Crítica, 15 (2), 271-282.

Martinelli, S. C. (2005). Construção de uma medida de avaliação da relação professor-aluno. Anais do /l Congresso Brasileiro de Avaliação Psicológica [CD]. Gramado: Instituto Brasileiro de Avaliação Psicológica.

McLeod, T. M. (1994). Social skills, school skills, and success in the high school: a comparison of teachers' and students' perceptions. Learning Disabilities Research and Practice, 9 (3), 142-147.

Meltzer, L., Katzir-Cohen, T., Miller, L., \& Roditi, B. (2001). The impact of effort and strategy use on academic performance: student and teacher perceptions. Learning Disability Quarterly, 24 (2), 85-98.

Morais, M. L. S., Otta, E., \& Scala, C. T. (2001). Status sociométrico e avaliação de características comportamentais: um estudo de competência social em pré-escolares. Psicologia: Reflexão e Crítica, 14 (1), 119-131.

Moreno, J. L. (1972). Fundamentos de la sociometría. Buenos Aires: Paidós.

Newcombe, N. (1999) Desenvolvimento infantil: abordagem de Mussen (8a. ed.). Porto Alegre: Artes Médicas.

Ray, G. E. (1995). Best friends networks of children across settings. Child Study Journal, 25 (3), 169-188.

Rolison, M. A., \& Medway, F. J. (1985). Teachers' expectations and attributions for student achievement: Effects of label performance pattern, and special education intervention. American Educational Research Journal, 22 (4), 561-573.

Rosenthal, R., \& Jacobson, L. (1968). Pygmalion in the classroom: Teacher expectation and pupil's intellectual development. New York: Holt, Rhinehat \& Winston.

Sabornie, E. (1987). Bi-directional social status of behaviorally disordered and nonhandicapped elementary school pupils. Behavioral Disorders, 13 (1), 45-57.

Saravali, E. G. (2004). As relações interpessoais dos alunos com problemas no aprendizado: um estudo a partir do 
referencial Moreniano. Revista Digital UMBRAL 2000, 16, disponível em www.reduc.cl.

Schiavoni, A., \& Martinelli, S. C. (2005). Percepção de alunos sobre as expectativas do professor acerca de seu desempenho: um estudo comparativo entre alunos com e sem dificuldades de aprendizagem. Interação em Psicologia, 9 (2), 311-319.

Sisto, F. F. (2003). Rejeição entre colegas e agressividade na infância. Cadernos de Psicologia, 13 (1), 83-98.

Sisto, F. F. (2005). Aceitação-rejeição para estudar e agressividade na escola. Psicologia em Estudo, 10 (1), 117-125.

Sisto, F. F., \& Martinelli, S. C. (2006). O papel das relações sociais na compreensão do fracasso escolar e das dificuldades de aprendizagem. In F.F. Sisto \& S.C. Martinelli (Orgs.), Afetividade e dificuldades de aprendizagem: uma abordagem psicopedagógica (pp.13-30). São Paulo: Vetor.
Sisto, F. F., Urquijo, S., \& Souza, M. T. C. C. (1999). Peer acceptance and cognitive development. Psychological Reports, 84 (2), 611-616.

Stiliadis, K., \& Wiener, J. (1989). Relationship between social perception and peer status in children with learning disabilities. Journal of Learning Disabilities, 22 (10), 624-629.

Stone, W. L., \& La Greca, A. M. (1990). The social status of children with learning disabilities: A reexamination. Journal of Learning Disabilities, 23 (1), 32-37.

Tonelotto, J. M. F. (2002). Aceitação e rejeição: percepção de escolares desatentos no ambiente escolar. Psicologia Escolar e Educacional, 6 (2), 141-148.

Recebido em: 7/3/2008

Versão final reapresentada em: 1/12/2008

Aprovado em: 5/3/2009 